Research Article

\title{
Positive solutions with exponential decay for a singular semipositone Fisher-like equation
}

\author{
Abdelhamid Benmezaï ${ }^{1, *}$, Nadir Benkaci-Ali ${ }^{2}$ \\ ${ }^{1}$ National Higher School of Mathematics, Sidi Abdallah, Algiers, Algeria \\ ${ }^{2}$ Faculty of Sciences, University of $M^{\prime}$ hmed Bouguerra, Boumerdes, Algeria
}

(Received: 17 November 2021. Received in revised form: 20 December 2021. Accepted: 22 December 2021. Published online: 25 December 2021.)

(c) 2021 the authors. This is an open access article under the CC BY (International 4.0) license (www.creativecommons.org/licenses/by/4.0/).

\section{Abstract}

This paper is concerned with the existence of positive solutions to the boundary value problem:

$$
\left\{\begin{array}{l}
-u^{\prime \prime}+c u^{\prime}+\lambda u=f(t, u(t)), t \in \mathbb{R}, \\
\lim _{t \rightarrow-\infty} e^{k|t|} u(t)=\lim _{t \rightarrow+\infty} e^{l|t|} u(t)=0,
\end{array}\right.
$$

where $\lambda$ and $c$ are positive constants, $k, l \in \mathbb{R}$, and $f: \mathbb{R} \times(0,+\infty) \rightarrow \mathbb{R}$ is a continuous function. The main existence result is proved by means of Guo-Krasnoselskii's fixed point theorem.

Keywords: Fisher-like equation; singular semipositone BVPs; fixed point theory in cones.

2020 Mathematics Subject Classification: 34B15, 34B16, 34B18, 34B40, 35K57.

\section{Introduction}

In this article, we deal with the existence of positive solutions to the boundary value problem (BVP for short):

$$
\left\{\begin{array}{l}
-u^{\prime \prime}+c u^{\prime}+\lambda u=f(t, u), t \in \mathbb{R}, \\
\lim _{t \rightarrow-\infty} e^{k|t|} u(t)=\lim _{t \rightarrow+\infty} e^{l|t|} u(t)=0,
\end{array}\right.
$$

where $\lambda$ and $c$ are positive constants, $k, l \in \mathbb{R}$, and $f: \mathbb{R} \times(0,+\infty) \rightarrow \mathbb{R}$ is a continuous function. By a positive solution to the BVP (1), we mean a function $u \in C^{2}(\mathbb{R})$ satisfying the ordinary differential equation in (1) such that $u(t)>0$ for all $t \in \mathbb{R}$ and $\lim _{t \rightarrow-\infty} e^{k|t|} u(t)=\lim _{t \rightarrow+\infty} e^{l|t|} u(t)=0$. Notice that if the constants $k$ and $l$ are positive, then by the boundary conditions in (1) we mean that we look for solutions having an exponential decay at $\pm \infty$.

The positivity of the solution $u$ is required here since the BVP (1) arises in the modeling of the propagation of wave fronts in combustion theory and epidemiology (see [2,9]), where $u$ stands for a concentration or a density. The positive constants $c$ and $\lambda$ refer to the wave speed of the front and the removal rate, respectively. The case where the BVP (1) is autonomous, that is $f(t, u)=f(u)$ with $f$ having a prescribed form, corresponds to the classical Fisher's equation.

There are many papers in the literature considering the case of the BVP (1) posed on the half-line, see [1,4-6,10-12] and references therein. However, to the best of authors' knowledge, there is no paper in the literature considering the singular semipositone case posed on the whole real line. Thus, the purpose of the present paper is to fill this gap.

Throughout this paper, we assume that

$$
\left\{\begin{array}{l}
\text { there exists a continuous function } q: \mathbb{R} \longrightarrow \mathbb{R}^{+} \text {such that } \int_{-\infty}^{+\infty} \eta(s) q(s) d s<\infty \\
\lim _{|t| \rightarrow \infty} p(t) q(t)=0 \text { and } \\
f(t, u)+q(t) \geq 0 \text { for all } t \in \mathbb{R} \text { and all } u>0
\end{array}\right.
$$

and

$$
\left\{\begin{array}{l}
\text { for all } \rho>0 \text { there exist functions } \omega_{\rho}, \Psi_{\rho}:(0,+\infty) \rightarrow(0,+\infty) \text { such that } \Psi_{\rho} \text { is nonincreasing, } \\
f\left(t, \frac{w}{p(t)}\right) \leq \omega_{\rho}(t) \Psi_{\rho}(w) \text { for all } t \in \mathbb{R} \text { and } w \in(0, \rho] \\
\lim _{t \rightarrow-\infty} q_{-}(t) \omega_{\rho}(t) \Psi_{\rho}(r \gamma(t))=\lim _{t \rightarrow+\infty} q_{+}(t) \omega_{\rho}(t) \Psi_{\rho}(r \gamma(t))=0 \text { and } \\
\int_{-\infty}^{+\infty} \delta(s) \omega_{\rho}(s) \Psi_{\rho}(r \gamma(s)) d s<\infty \text { for all } r \in(0, \rho]
\end{array}\right.
$$

*Corresponding author (aehbenmezai@gmail.com). 
where

$$
\begin{aligned}
& p(t)=e^{-r_{2}|t|}, \\
& q_{-}(t)=\max \left(p(t), e^{k|t|}\right)=\exp \left(\max \left(k,-r_{2}\right)|t|\right), \\
& q_{+}(t)=\max \left(p(t), e^{l|t|}\right)=\exp \left(\max \left(l,-r_{2}\right)|t|\right), \\
& \gamma(t)=\min \left(e^{2 r_{2} t}, e^{\left(r_{1}-r_{2}\right) t}\right), \\
& \widetilde{\gamma}(t)=\frac{\gamma(t)}{p(t)}=\min \left(e^{r_{1} t}, e^{r_{2} t}\right), \\
& \delta(t)=\min \left(e^{-r_{1} t}, e^{-r_{2} t}\right)=\left(\max \left(e^{r_{1} t}, e^{r_{2} t}\right)\right)^{-1}, \\
& \eta(t)=\max \left(e^{-r_{1} t}, e^{-r_{2} t}\right),
\end{aligned}
$$

$r_{1}$ and $r_{2}$ are the solutions of the characteristic equation $-X^{2}+c X+\lambda=0$ with $r_{1}<0<r_{2}$.

Remark 1.1. Notice that in the case where $k, l \geq 0$, we have

$$
q_{-}(t)=e^{k|t|}, q_{+}(t)=e^{l|t|}
$$

and so

$$
\lim _{t \rightarrow-\infty} e^{k|t|} \omega_{\rho}(t) \Psi_{\rho}(r \gamma(t))=\lim _{t \rightarrow+\infty} e^{l|t|} \omega_{\rho}(t) \Psi_{\rho}(r \gamma(t))=0
$$

implies that

$$
\int_{-\infty}^{+\infty} \delta(s) \omega_{\rho}(s) \Psi_{\rho}(r \gamma(s)) d s<\infty
$$

In such a situation Hypothesis (3) can be relaxed to

$$
\left\{\begin{array}{l}
\text { for all } \rho>0 \text { there exist functions } \omega_{\rho}, \Psi_{\rho}:(0,+\infty) \rightarrow(0,+\infty) \\
\text { such that } \Psi_{\rho} \text { is nonincreasing, } \\
\left|f\left(t, \frac{w}{p(t)}\right)\right| \leq \omega_{\rho}(t) \Psi_{\rho}(w) \text { for all } t \in \mathbb{R} \text { and all } w \in(0, \rho], \\
\lim _{t \rightarrow-\infty} e^{k|t|} \omega_{\rho}(t) \Psi_{\rho}(r \gamma(t))=\lim _{t \rightarrow+\infty} e^{l|t|} \omega_{\rho}(t) \Psi_{\rho}(r \gamma(t))=0 \text { for all } r \in(0, \rho] .
\end{array}\right.
$$

Remark 1.2. Hypothesis (3) cover the case of the BVP(1) where the nonlinearity $f$ satisfies the polynomial growth condition

$$
|f(t, u)| \leq a(t)+b(t) u^{\sigma}
$$

where $\sigma>1$ and $a, b \in C(\mathbb{R})$ are such that

$$
\begin{aligned}
\lim _{t \rightarrow-\infty} q_{-}(t) a(t)=\lim _{t \rightarrow+\infty} q_{+}(t) a(t)= & \lim _{t \rightarrow-\infty} q_{-}(t) b(t)(p(t))^{-\sigma}=\lim _{t \rightarrow+\infty} q_{+}(t) b(t)(p(t))^{-\sigma}=0, \\
& \int_{-\infty}^{+\infty} \delta(s) a(s) d s<\infty
\end{aligned}
$$

and

$$
\int_{-\infty}^{+\infty} \delta(s) b(s)(p(s))^{-\sigma} d s<\infty
$$

To see this, one has to take

$$
\omega_{\rho}(t)=\max \left(a(t), b(t)(p(t))^{-\sigma}\right) \text { for all } \rho>0 .
$$

Notice that satisfying Hypothesis (3), the nonlinearity $f$ may exhibit singular at $u=0$. It is well known that the BVP (1) is called positone if $q(t)=0$ for all $t \in \mathbb{R}$, and semipositone if $q\left(t_{0}\right)>0$ for some $t_{0} \in \mathbb{R}$. Study of existence of positive solutions for semipositone BVPs still attract the attention of many researchers (see for instance, [7, 8] and references therein).

Our approach in this work is based on a fixed point formulation and we will use the Guo-Krasnoselskii's fixed point theorem. So, let us present some basic background related to this principle.

Let $(E,\|\|$.$) be a real Banach space. A nonempty closed convex subset C$ of $E$ is said to be a cone in $E$ if $C \cap(-C)=\left\{0_{E}\right\}$ and $t C \subset C$ for all $t \geq 0$.

Let $\Omega$ be a nonempty subset in $E$. A mapping $A: \Omega \rightarrow E$ is said to be compact if it is continuous and $A(\Omega)$ is relatively compact in $E$. 
The main tool of this work is the following Guo-Krasnoselskii's fixed point theorem.

Theorem 1.1. Let $P$ be a cone in $E$ and let $\Omega_{1}, \Omega_{2}$ be bounded open subsets of $E$ with $0 \in \Omega_{1}$ and $\bar{\Omega}_{1} \subset \Omega_{2}$. If

$$
T: P \cap\left(\bar{\Omega}_{2} \backslash \Omega_{1}\right) \rightarrow P
$$

is a compact mapping such that either:

1. $\|T u\| \leq\|u\|$ for $u \in P \cap \partial \Omega_{1}$ and $\|T u\| \geq\|u\|$ for $u \in P \cap \partial \Omega_{2}$, or

2. $\|T u\| \geq\|u\|$ for $u \in P \cap \partial \Omega_{1}$ and $\|T u\| \leq\|u\|$ for $u \in P \cap \partial \Omega_{2}$,

Then $T$ has at least one fixed point in $P \cap\left(\bar{\Omega}_{2} \backslash \Omega_{1}\right)$.

The paper is organized as follows. The next section is devoted to the fixed point formulation of the BVP (1). In Section 3 , we present the main existence result of this paper. We end the paper by giving an illustrative example.

\section{Fixed point formulation}

We start this section by the following important lemma. It will propose a cone in a specific functional Banach space, favorable to the use of Theorem 1.1. Let $G: \mathbb{R} \times \mathbb{R} \rightarrow \mathbb{R}^{+}$be the function defined by

$$
G(t, s)=\frac{1}{r_{2}-r_{1}}\left\{\begin{array}{l}
\exp \left(r_{1}(t-s)\right), \text { if } s \leq t \\
\exp \left(r_{2}(t-s)\right), \text { if } t \leq s .
\end{array}\right.
$$

Lemma 2.1. The function $G$ has the following properties:

(i). $0<G(t, s) \leq \frac{1}{r_{2}-r_{1}}$ for all $t, s \in \mathbb{R}$.

(ii). For all $t, \tau, s \in \mathbb{R}$, the following inequality holds

$$
p(t) G(t, s) \geq \gamma(t) p(\tau) G(\tau, s) .
$$

(iii). Let $h: \mathbb{R} \longrightarrow \mathbb{R}^{+}$be a measurable function. If $\delta h \in L^{1}(\mathbb{R})$ then for all $t \in \mathbb{R}$, the following inequality holds

$$
\int_{-\infty}^{+\infty} G(t, s) h(s) d s \leq \frac{1}{\left(r_{2}-r_{1}\right) \delta(t)} \int_{-\infty}^{+\infty} \delta(s) h(s) d s .
$$

Proof. Part (i) is obvious. For proving Part (ii), we distinguish four cases. Set $Q(t, \tau, s)=\frac{p(t) G(t, s)}{p(\tau) G(\tau, s)}$.

Case (a). $\tau, t \geq 0$. In this case, we have

$$
Q(t, \tau, s)=\left\{\begin{array}{l}
\exp \left(-\left(r_{2}-r_{1}\right) t+\left(r_{2}-r_{1}\right) \tau\right) \geq e^{-\left(r_{2}-r_{1}\right) t}, \text { if } s \leq \tau \leq t \\
\exp \left(-\left(r_{2}-r_{1}\right) t+\left(r_{2}-r_{1}\right) s\right) \geq e^{-\left(r_{2}-r_{1}\right) t}, \text { if } \tau \leq s \leq t \\
1, \text { if } \tau \leq t \leq s \\
\exp \left(-\left(r_{2}-r_{1}\right) t+\left(r_{2}-r_{1}\right) \tau\right) \geq e^{-\left(r_{2}-r_{1}\right) t}, \text { if } s \leq t \leq \tau, \\
\exp \left(\left(r_{2}-r_{1}\right) \tau-\left(r_{2}-r_{1}\right) s\right) \geq 1, \text { if } t \leq s \leq \tau, \\
1, \text { if } t \leq \tau \leq s
\end{array}\right.
$$

Thus, $Q(t, \tau, s) \geq \gamma(t)$.

Case (b). $\tau, t \leq 0$. In this case, we have

$$
Q(t, \tau, s)=\left\{\begin{array}{l}
\exp \left(\left(r_{2}+r_{1}\right) t-\left(r_{2}+r_{1}\right) \tau\right) \geq e^{\left(r_{2}+r_{1}\right) t}, \text { if } s \leq \tau \leq t, \\
\exp \left(-\left(r_{2}-r_{1}\right) t-2 r_{2} \tau+\left(r_{2}-r_{1}\right) s\right) \geq e^{-\left(r_{2}-r_{1}\right) t}, \text { if } \tau \leq s \leq t, \\
\exp \left(2 r_{2} t-2 r_{2} \tau\right) \geq e^{2 r_{2} t}, \text { if } \tau \leq t \leq s, \\
\exp \left(\left(r_{2}+r_{1}\right) t-\left(r_{2}+r_{1}\right) \tau\right) \geq e^{\left(r_{2}+r_{1}\right) t}, \text { if } s \leq t \leq \tau, \\
\exp \left(2 r_{2} t-\left(r_{2}+r_{1}\right) \tau-\left(r_{2}-r_{1}\right) s\right) \geq e^{2 r_{2} t}, \text { if } t \leq s \leq \tau, \\
\exp \left(2 r_{2} t-2 r_{2} \tau\right) \geq e^{2 r_{2} t}, \text { if } t \leq \tau \leq s
\end{array}\right.
$$


Hence, $Q(t, \tau, s) \geq \gamma(t)$.

Case (c). $\tau \leq 0, t \geq 0$. In this case, we have

$$
Q(t, \tau, s)=\left\{\begin{array}{l}
\exp \left(-\left(r_{2}-r_{1}\right) t-\left(r_{2}+r_{1}\right) \tau\right) \geq e^{-\left(r_{2}-r_{1}\right) t}, \text { if } s \leq \tau \leq t \\
\exp \left(-\left(r_{2}-r_{1}\right) t-2 r_{2} \tau+\left(r_{2}-r_{1}\right) s\right) \geq e^{-\left(r_{2}-r_{1}\right) t}, \text { if } \tau \leq s \leq t \\
\exp \left(-2 r_{2} \tau\right) \geq 1, \text { if } \tau \leq t \leq s
\end{array}\right.
$$

Therefore, $Q(t, \tau, s) \geq \gamma(t)$.

Case (d). $\tau \geq 0, t \leq 0$. In this case, we have

$$
Q(t, \tau, s)=\left\{\begin{array}{l}
\exp \left(\left(r_{2}+r_{1}\right) t+\left(r_{2}-r_{1}\right) \tau\right) \geq e^{\left(r_{2}+r_{1}\right) t}, \text { if } s \leq t \leq \tau, \\
\exp \left(2 r_{2} t+\left(r_{2}-r_{1}\right) \tau-\left(r_{2}-r_{1}\right) s\right) \geq e^{2 r_{2} t}, \text { if } t \leq s \leq \tau, \\
\exp \left(2 r_{2} t\right), \text { if } t \leq \tau \leq s
\end{array}\right.
$$

Thus, $Q(t, \tau, s) \geq \gamma(t)$. Finally, we prove Part (iii). Let $h: \mathbb{R} \longrightarrow \mathbb{R}^{+}$be a function such that $\delta h \in L^{1}(\mathbb{R})$. For all $t \geq 0$ (the case $t \leq 0$ is obtained similarly) we have

$$
\begin{aligned}
\left(r_{2}-r_{1}\right) \int_{-\infty}^{+\infty} G(t, s) h(s) d s & =e^{r_{1} t} \int_{-\infty}^{0} e^{-r_{1} s} h(s) d s+e^{r_{1} t} \int_{0}^{t} e^{-r_{1} s} h(s) d s+e^{r_{2} t} \int_{t}^{+\infty} e^{-r_{2} s} h(s) d s \\
& =e^{r_{1} t} \int_{-\infty}^{0} \delta(s) h(s) d s+e^{r_{2} t} \int_{t}^{+\infty} \delta(s) h(s) d s+e^{r_{1} t} \int_{0}^{t} e^{\left(r_{2}-r_{1}\right) s} \delta(s) h(s) d s \\
& \leq e^{r_{1} t} \int_{-\infty}^{0} \delta(s) h(s) d s+e^{r_{2} t} \int_{t}^{+\infty} \delta(s) h(s) d s+e^{r_{2} t} \int_{0}^{t} \delta(s) h(s) d s \\
& \leq \max \left(e^{r_{1} t}, e^{r_{2} t}\right) \int_{-\infty}^{+\infty} \delta(s) h(s) d s=\frac{1}{\delta(t)} \int_{-\infty}^{+\infty} \delta(s) h(s) d s
\end{aligned}
$$

This completes the proof.

It is well known that the use of Theorem 1.1 requires the positivity. Since the nonlinearity $f$ is not positive, we will make on the BVP (1) a translation $v=u+\phi$ where the associated modified problem has a positive nonlinearity. The following lemma provides such a function $\phi$.

Lemma 2.2. Assume that Hypothesis (2) holds and let $\phi$ be the function defined by

$$
\phi(t)=\int_{-\infty}^{+\infty} G(t, s) q(s) d s \text { for all } t \in \mathbb{R} .
$$

Then

$$
\phi^{*}=\sup _{t \in \mathbb{R}}\left(\frac{\phi(t)}{\widetilde{\gamma}(t)}\right)<\infty .
$$

Proof. For all $t \geq 0$, we have

$$
\begin{aligned}
\frac{\phi(t)}{\widetilde{\gamma}(t)} & =\frac{1}{r_{2}-r_{1}}\left(\frac{e^{r_{1} t} \int_{-\infty}^{t} e^{-r_{1} s} q(s) d s}{e^{r_{1} t}}+\frac{e^{r_{2} t} \int_{t}^{+\infty} e^{-r_{2} s} q(s) d s}{e^{r_{1} t}}\right) \\
& \leq \frac{1}{r_{2}-r_{1}}\left(\int_{-\infty}^{+\infty} \eta(s) q(s) d s+\frac{e^{r_{2} t} e^{\left(r_{1}-r_{2}\right) t} \int_{t}^{+\infty} e^{-r_{1} s} q(s) d s}{e^{r_{1} t}}\right) \\
& \leq \frac{2}{r_{2}-r_{1}} \int_{-\infty}^{+\infty} \eta(s) q(s) d s .
\end{aligned}
$$

Similarly, for all $t \leq 0$ we have

$$
\begin{aligned}
\frac{\phi(t)}{\widetilde{\gamma}(t)} & \leq \frac{1}{r_{2}-r_{1}}\left(\frac{e^{r_{1} t} e^{\left(r_{2}-r_{1}\right) t} \int_{-\infty}^{t} e^{-r_{2} s} q(s) d s}{e^{r_{2} t}}+\int_{t}^{+\infty} e^{-r_{2} s} q(s) d s\right) \\
& \leq \frac{2}{r_{2}-r_{1}} \int_{-\infty}^{+\infty} \eta(s) q(s) d s .
\end{aligned}
$$

This completes the proof. 
The functional framework in which we will solve the BVP (1) consists in the following Banach space $E$ and the cone $P$ given below and suggested by Lemma 2.1. In the remaining part of this paper, let $E$ be the linear space defined by

$$
E=\left\{u \in C(\mathbb{R}, \mathbb{R}): \lim _{|t| \rightarrow \infty} p(t) u(t)=0\right\} .
$$

Equipped with the norm $\|\cdot\|$, where for $u \in E\|u\|=\sup _{t \in \mathbb{R}}(p(t)|u(t)|), E$ becomes a Banach space. The subset $P$ of $E$ given by

$$
P=\{u \in E: u(t) \geq \widetilde{\gamma}(t)\|u\| \text { for all } t \in \mathbb{R}\}
$$

is a cone of $E$.

The following lemma is an adapted version to the case of the linear space $E$ of Corduneanu's compactness criterion (see [3], p. 62). This lemma will be used to prove that the operator in the fixed point formulation of the BVP (1) is compact.

Lemma 2.3. A nonempty subset $M$ of $E$ is relatively compact if the following conditions hold:

(a) $M$ is bounded in $E$,

(b) the set $\{u: u(t)=p(t) x(t), x \in M\}$ is locally equicontinuous on $\mathbb{R}$, and

(c) the set $\{u: u(t)=p(t) x(t), x \in M\}$ is equiconvergent at $\pm \infty$.

Lemma 2.4. Assume that Hypotheses (2) and (3) hold $k<-r_{1}$ and $l<r_{2}$. Then for all real numbers $r$ and $R$ with $R>r>\phi^{*}$ there exists a compact operator $T_{r, R}: P_{r, R} \rightarrow P$, where $P_{r, R}=P \cap\left(\bar{B}(0, R) \backslash(B(0, r))\right.$ such that if $v$ is a fixed point of $T_{r, R}$ then $u=v-\phi$ is a positive solution to the BVP (1).

Proof. Let $r, R$ be real numbers such that $R>r>\phi^{*}$ and let $\Phi$ be the function defined by

$$
\Phi(s)=\omega_{R}(s) \Psi_{R}\left(\left(r-\phi^{*}\right) \gamma(s)\right)
$$

where $\omega_{R}$ and $\Psi_{R}$ are the functions given by Hypothesis (3) for $\rho=R$ and notice that for all $u \in P_{r, R}$ and all $t \in \mathbb{R}$, we have

$$
f(t, u(t)-\phi(t))+q(t) \leq \Phi(t)+q(t)
$$

The proof is divided into three steps.

Step 1. In this step we prove the existence of the operator $T_{r, R}$. To this aim let $u \in P_{r, R}$, for all $t \in \mathbb{R}$ we have from Assertion (iii) in Lemma 2.1 and Hypothesis (3),

$$
\begin{aligned}
\int_{-\infty}^{+\infty} G(t, s)(f(s, u(s)-\phi(s))+q(s)) d s & \leq \int_{-\infty}^{+\infty} G(t, s)(\Phi(s)+q(s)) d s \\
& \leq \frac{1}{\left(r_{2}-r_{1}\right) \delta(t)} \int_{-\infty}^{+\infty} \delta(s)(\Phi(s)+q(s)) d s<\infty .
\end{aligned}
$$

Thus, let $v$ be the function defined by

$$
v(t)=\int_{-\infty}^{+\infty} G(t, s)(f(s, u(s)-\phi(s))+q(s)) d s .
$$

Clealy, $v$ is continuous on $\mathbb{R}$ and $v(t)>0$ for all $t \in \mathbb{R}$. Moreover, we have

$$
p(t) v(t) \leq \frac{1}{\left(r_{2}-r_{1}\right)}\left(J_{1}(t)+J_{2}(t)\right),
$$

where

$$
J_{1}(t)=\frac{\int_{-\infty}^{t} e^{-r_{1} s}(\Phi(s)+q(s)) d s}{\exp \left(r_{2}|t|-r_{1} t\right)} \text { and } J_{2}(t)=\frac{\int_{t}^{+\infty} e^{-r_{2} s}(\Phi(s)+q(s)) d s}{\exp \left(r_{2}|t|-r_{2} t\right)}
$$

Since for $t \leq 0$,

$$
J_{1}(t) \leq \int_{-\infty}^{t} \delta(s)(\Phi(s)+q(s)) d s
$$

and for $t \geq 0$,

$$
J_{2}(t)=\int_{t}^{+\infty} \delta(s)(\Phi(s)+q(s)) d s
$$


we obtain from Hypotheses (2) and (3) that $\lim _{t \rightarrow-\infty} J_{1}(t)=\lim _{t \rightarrow+\infty} J_{2}(t)=0$. Now, applying L'Hopital's rule, we obtain from Hypotheses (2) and (3) that

$$
\lim _{t \rightarrow+\infty} J_{1}(t)=\lim _{t \rightarrow+\infty} \frac{e^{-r_{1} t}(\Phi(t)+q(t))}{\left(r_{2}-r_{1}\right) \exp \left(\left(r_{2}-r_{1}\right) t\right)}=\frac{1}{\left(r_{2}-r_{1}\right)} \lim _{t \rightarrow+\infty} p(t)(\Phi(t)+q(t))=0
$$

and

$$
\lim _{t \rightarrow-\infty} J_{2}(t)=\lim _{t \rightarrow-\infty} \frac{e^{-r_{2} t}(\Phi(t)+q(t))}{2 r_{2} \exp \left(-2 r_{2} t\right)}=\frac{1}{2 r_{2}} \lim _{t \rightarrow-\infty} p(t)(\Phi(t)+q(t))=0 .
$$

Hence, we conclude that $\lim _{|t| \rightarrow+\infty} p(t) v(t)=0$ and $v \in E$.

Assertion (ii) in Lemma 2.1 leads to

$$
\begin{aligned}
p(t) v(t) & =\int_{-\infty}^{+\infty} p(t) G(t, s)(f(s, u(s)-\phi(s))+q(s)) d s \\
& \geq \gamma(t) \int_{-\infty}^{+\infty} p(\tau) G(\tau, s)(f(s, u(s)-\phi(s))+q(s)) d s \\
& \geq \gamma(t) v(\tau)
\end{aligned}
$$

for all $t, \tau \in \mathbb{R}$. Passing to the supremum on $\tau$ yields

$$
v(t) \geq \widetilde{\gamma}(t)\|v\|,
$$

proving that $v \in P$ and the operator $T_{r, R}: P_{r, R} \rightarrow P$ where for $u \in P_{r, R}$,

$$
T_{r, R} u(t)=\int_{-\infty}^{+\infty} G(t, s)(f(s, u(s)-\phi(s))+q(s)) d s
$$

is well defined.

Step 2. In this step, we prove that the operator $T_{r, R}$ is compact. Let $\left(u_{n}\right)$ be a sequence in $P_{r, R}$ such that $\lim _{n \rightarrow \infty} u_{n}=u$ in $E$ given by Hypothesis (3), then for all $n \geq 1$ we have

$$
\begin{aligned}
\left\|T_{r, R} u_{n}-T_{r, R} u\right\| & =\sup _{t \geq 0}\left(p(t)\left|T_{r, R} u_{n}(t)-T_{r, R} u(t)\right|\right) \\
& \leq \sup _{t \geq 0}\left(\frac{p(t)}{\left(r_{2}-r_{1}\right) \delta(t)} \int_{0}^{+\infty} \delta(s) \mid f\left(s, u_{n}(s)-\phi(s)\right)-f((s, u(s)-\phi(s)) \mid d s)\right. \\
& \leq \frac{1}{\left(r_{2}-r_{1}\right)} \int_{0}^{+\infty} \delta(s) \mid f\left(s, u_{n}(s)-\phi(s)\right)-f((s, u(s)-\phi(s)) \mid d s .
\end{aligned}
$$

Because of

$$
\mid f\left(s, u_{n}(s)-\phi(s)\right)-f((s, u(s)-\phi(s)) \mid \rightarrow 0, \text { as } n \rightarrow+\infty
$$

for all $s>0$ and

$$
\delta(s) \mid f\left(s, u_{n}(s)-\phi(s)\right)-f((s, u(s)-\phi(s)) \mid \leq 2 \delta(s) \Phi(s)
$$

with

$$
\int_{-\infty}^{+\infty} \delta(s) \Phi(s) d s<\infty,
$$

the Lebesgue dominated convergence theorem guarantees that $\lim _{n \rightarrow \infty}\left\|T_{r, R} u_{n}-T_{r, R} u\right\|=0$. Hence, we have proved that $T_{r, R}$ is continuous. Also, for all $u \in P_{r, R}$, we have

$$
\begin{aligned}
\left\|T_{r, R} u\right\| & \leq \sup _{t \geq 0}\left(\frac{p(t)}{\left(r_{2}-r_{1}\right) \delta(t)} \int_{0}^{+\infty} \delta(s)(\Phi(s)+q(s)) d s\right) \\
& \leq \frac{1}{r_{2}-r_{1}} \int_{0}^{+\infty} \delta(s)(\Phi(s)+q(s)) d s<\infty
\end{aligned}
$$

proving that $T_{r, R}\left(P_{r, R}\right)$ is bounded in $E$. 
Next, let $t_{1}, t_{2} \in[\alpha, \beta] \subset \mathbb{R}$, for all $u \in P_{r, R}$ then we have

$$
\begin{aligned}
\left|p\left(t_{2}\right) T_{r, R} u\left(t_{2}\right)-p\left(t_{1}\right) T_{r, R} u\left(t_{1}\right)\right| \leq & \left|p_{1}\left(t_{2}\right)-p_{1}\left(t_{1}\right)\right| \int_{-\infty}^{\zeta} e^{-r_{1} s}(\Phi(s)+q(s)) d s+\left|p_{2}\left(t_{2}\right)-p_{2}\left(t_{1}\right)\right| \int_{\eta}^{+\infty} e^{-r_{2} s}(\Phi(s)+q(s)) d s \\
& +C_{\eta, \zeta} \int_{t_{1}}^{t_{2}}(\Phi(s)+q(s)) d s
\end{aligned}
$$

where for $i=1,2, p_{i}(t)=e^{-r_{2}|t|+r_{i} t}$ and

$$
C_{\eta, \zeta}=2 \sup _{t, s \in[\eta, \zeta]} p(t) G(t, s) .
$$

Because $p_{1}, p_{2}$, and $t \rightarrow \int_{0}^{t}(\Phi(s)+q(s)) d s$ are uniformly continuous on compact intervals, the above estimate proves that $T_{r, R}$ is equicontinuous on compact intervals. Furthermore, for all $u \in T_{r, R}$ and $t \in \mathbb{R}$, we have

$$
p(t) T_{r, R} u(t) \leq p(t) \int_{-\infty}^{+\infty} G(t, s)(\Phi(s)+q(s)) d s=H(t) .
$$

By means of L'Hopital's rule, we obain from Hypotheses (2) and (3) that

$$
\lim _{|t| \rightarrow \infty} H(t)=\lim _{|t| \rightarrow \infty} p(t)(\Phi(t)+q(t))=0,
$$

proving the equiconvergence of $T_{r, R}$. Therefore, in view of Lemma 2.3, $T_{r, R}$ is relatively compact in $E$.

Step 3. In this step, we prove that if $v \in P_{r, R}$ is a fixed points of $T_{r, R}$, then $u=v-\phi$ is a positive solution to the BVP (1). Let $v \in P_{r, R}$ be a fixed point of $T_{r, R}$ and let $u=v-\phi$. For all $t \in \mathbb{R}$ we have

$$
u(t)+\phi(t)=u(t)+\int_{-\infty}^{+\infty} G(t, s) q(s) d s=\int_{-\infty}^{+\infty} G(t, s)(f(s, u(s))+q(s)) d s
$$

leading to

$$
\begin{aligned}
u(t) & =\int_{-\infty}^{+\infty} G(t, s) f(s, u(s)) d s \\
& =\frac{1}{r_{2}-r_{1}}\left(e^{r_{1} t} \int_{-\infty}^{t} e^{-r_{1} s} f(s, u(s)) d s+e^{r_{2} t} \int_{t}^{+\infty} e^{-r_{2} s} f(s, u(s)) d s\right), \\
u^{\prime}(t) & =\frac{r_{1} e^{r_{1} t}}{r_{2}-r_{1}} \int_{-\infty}^{t} e^{-r_{1} s} f(s, u(s)) d s+\frac{r_{2} e^{r_{2} t}}{r_{2}-r_{1}} \int_{t}^{+\infty} e^{-r_{2} s} f(s, u(s)) d s \text { and } \\
u^{\prime \prime}(t) & =\frac{\left(r_{1}\right)^{2} e^{r_{1} t}}{r_{2}-r_{1}} \int_{-\infty}^{t} e^{-r_{1} s} f(s, u(s)) d s+\frac{\left(r_{2}\right)^{2} e^{r_{2} t}}{r_{2}-r_{1}} \int_{t}^{+\infty} e^{-r_{2} s} f(s, u(s)) d s-f(t, u(t)) .
\end{aligned}
$$

Thus, we obtain

$$
\begin{aligned}
-u^{\prime \prime}(t)+c u^{\prime}(t)+\lambda u(t) & =\frac{-r_{1}^{2}+c r_{1}+\lambda}{r_{2}-r_{1}} \int_{-\infty}^{t} G(t, s) f(s, u(s)) d s+\frac{-r_{2}^{2}+c r_{2}+\lambda}{r_{2}-r_{1}} \int_{t}^{+\infty} G(t, s) f(s, u(s)) d s+f(t, u(t)) \\
& =f(t, u(t)) .
\end{aligned}
$$

Now, we need to prove that $u$ satisfies the boundary conditions: $\lim _{t \rightarrow-\infty} e^{l|t|} u(t)=\lim _{t \rightarrow+\infty} e^{k|t|} u(t)=0$. We have

$$
e^{l|t|} u(t) \leq \frac{1}{r_{2}-r_{1}}\left(L_{1}(t)+L_{2}(t)\right)
$$

and

where

$$
e^{k|t|} u(t) \leq \frac{1}{r_{2}-r_{1}}\left(K_{1}(t)+K_{2}(t)\right)
$$

$$
\begin{gathered}
L_{1}(t)=\frac{\int_{-\infty}^{t} e^{-r_{1} s} \Phi(s) d s}{\exp \left(-l|t|-r_{1} t\right)}, \quad L_{2}(t)=\frac{\int_{t}^{+\infty} e^{-r_{2} s} \Phi(s) d s}{\exp \left(-l|t|-r_{2} t\right)}, \\
K_{1}(t)=\frac{\int_{-\infty}^{t} e^{-r_{1} s} \Phi(s) d s}{\exp \left(-k|t|-r_{1} t\right)} \text { and } K_{2}(t)=\frac{\int_{t}^{+\infty} e^{-r_{2} s} \Phi(s) d s}{\exp \left(-k|t|-r_{2} t\right)} .
\end{gathered}
$$


Since for $t \leq 0$,

$$
L_{1}(t) \leq\left\{\begin{array}{l}
\int_{-\infty}^{t} \delta(s) \Phi(s) d s, \text { if } l \leq r_{1} \\
\frac{\int_{-\infty}^{t} \delta(s) \Phi(s) d s}{\exp \left(\left(l-r_{1}\right) t\right)}, \text { if } l>r_{1}
\end{array}\right.
$$

and for $t \geq 0$,

$$
K_{2}(t) \leq\left\{\begin{array}{l}
\int_{t}^{+\infty} \delta(s) \Phi(s) d s, \text { if } k \leq-r_{2}, \\
\frac{\int_{t}^{+\infty} \delta(s) \Phi(s) d s}{\exp \left(-\left(k+r_{2}\right) t\right)}, \text { if } k>-r_{2},
\end{array}\right.
$$

Hypothesis (3) and L'Hopital's rule lead to

$$
\lim _{t \rightarrow-\infty} L_{1}(t)=\lim _{t \rightarrow+\infty} K_{2}(t)=0 .
$$

Taking into account the conditions $k<-r_{1}$ and $l<r_{2}$ and Hypothesis (3), the L'Hopital's rule leads to

$$
\lim _{t \rightarrow-\infty} L_{2}(t)=\lim _{t \rightarrow-\infty} \frac{-e^{-r_{2} t} \Phi(s)}{\left(l-r_{2}\right) \exp \left(\left(l-r_{2}\right) t\right)}=\frac{1}{r_{2}-l} \lim _{t \rightarrow-\infty} e^{l|t|} \Phi(s)=0
$$

and

$$
\lim _{t \rightarrow+\infty} K_{1}(t)=\lim _{t \rightarrow+\infty} \frac{e^{-r_{1} t} \Phi(s)}{-\left(k+r_{1}\right) \exp \left(-\left(k+r_{1}\right) t\right)}=\frac{-1}{\left(k+r_{1}\right)} \lim _{t \rightarrow+\infty} e^{k|t|} \Phi(s)=0 .
$$

Hence, we have proved that

$$
\lim _{t \rightarrow-\infty} e^{l|t|} u(t)=\lim _{t \rightarrow+\infty} e^{k|t|} u(t)=0,
$$

completing the proof of the lemma.

\section{Main result}

Before proving the main result of this paper, we first introduce the following notations. Let

$$
L_{\delta}^{1}(\mathbb{R})=\left\{m: \mathbb{R} \longrightarrow \mathbb{R}^{+} \text {measurable: } \int_{-\infty}^{+\infty} \delta(s) m(s) d s<\infty\right\}
$$

and for $\alpha \in L_{\delta}^{1}(\mathbb{R})$ and $\theta>0$, take

$$
\Gamma(\alpha)=\sup _{t \in \mathbb{R}}\left(p(t) \int_{-\infty}^{+\infty} G(t, s) \alpha(s) d s\right)
$$

and

$$
\Delta(\alpha, \theta)=\sup _{t \in \mathbb{R}}\left(p(t) \int_{-\theta}^{\theta} G(t, s) \alpha(s) d s\right) .
$$

Theorem 3.1. Suppose that Hypotheses (2) and (3) hold, $k<-r_{1}, l<r_{2}$, and

(a). there exist a function $\alpha \in L_{\delta}^{1}(\mathbb{R})$ and $R_{1}>\max \left(\phi^{*}, \Gamma(\alpha)\right)$ such that

$$
f\left(t, \frac{u}{p(t)}\right)+q(t) \leq \alpha(t)
$$

for all $t \in \mathbb{R}$ and $u \in\left(0, R_{1}\right)$;

(b). there exist $\theta>0$, a function $\beta \in L_{\delta}^{1}(\mathbb{R})$ and $R_{2} \in\left(\phi^{*}, \Delta(\beta, \theta)\right)$ with $R_{2} \neq R_{1}$ such that

$$
f\left(t, \frac{u}{p(t)}\right)+q(t) \geq \beta(t),
$$

for all $t \in[-\theta, \theta]$, all $u \in\left[\gamma_{\sigma}\left(R_{2}-\phi^{*}\right), R_{2}\right]$ where $\gamma_{\sigma}=\min _{s \in[-\theta, \theta]} \gamma(s)$.

Then, the BVP (1) admits a positive solution. 
Proof. Without loss of generality, assume that $R_{1}<R_{2}$ and let $T=T_{R_{1}, R_{2}}$ be the operator given by Lemma 2.4. The following estimates hold, for all $v \in P \cap \partial B\left(0, R_{1}\right)$ and all $t \in \mathbb{R}$,

$$
\begin{aligned}
p(t) T v(t) & =p(t) \int_{-\infty}^{+\infty} G(t, s)(f(s, v(s)-\phi(s))+q(s)) d s \\
p(t) T v(t) & \leq p(t) \int_{-\infty}^{+\infty} G(t, s) \alpha(s) d s \\
& \leq \sup _{t>0}\left(p(t) \int_{-\infty}^{+\infty} G(t, s) \alpha(s) d s\right)=\Gamma(\alpha) .
\end{aligned}
$$

Passing to the supremum in the above estimates, we get

$$
\|T v\| \leq \Gamma(\alpha) \leq R_{1}=\|v\| .
$$

For all $v \in P \cap \partial B\left(0, R_{2}\right)$ and $s \in[-\theta, \theta]$,

$$
R_{2} \geq(v(s)-\phi(s)) p(s) \geq\left(R_{2}-\phi^{*}\right) \gamma(s)=\left(R_{2}-\phi^{*}\right) \gamma_{\theta}
$$

Assumption (b) and (4) lead to the following estimates

$$
\begin{aligned}
\|T v\| & \geq \sup _{t \in \mathbb{R}}\left(p(t) \int_{-\theta}^{\theta} G(t, s)(f(s,(v(s)-\phi(s)))+q(s)) d s\right) \\
& \geq \sup _{t \in \mathbb{R}}\left(p(t) \int_{-\theta}^{\theta} G(t, s) \beta(s) d s\right) \\
& =\Delta(\beta, \theta) \geq R_{2}=\|v\| .
\end{aligned}
$$

Thus, it follows from Theorem 1.1 that $T_{R_{1}, R_{2}}$ admits a fixed point $v$ such that $R_{1} \leq\|v\| \leq R_{2}$. Then, by Lemma 2.4, $u=v-\phi$ is a positive solution to the BVP (1).

For $\theta>0$, take

$$
f_{\theta}=\liminf _{w \rightarrow+\infty}\left(\min _{t \in[-\theta, \theta]} \frac{f\left(t, \frac{w}{p(t)}\right)}{w}\right) .
$$

We obtain from Theorem 3.1 the next corollary.

Corollary 3.1. Suppose that Hypotheses (2) and (3) hold, $k<-r_{1}$ and

(c). there exists $R_{1}>\phi^{*}$ such that $\Gamma\left(\alpha_{1}\right)<R_{1}$ where

$$
\alpha_{1}=\omega_{R_{1}}(s) \Psi_{R_{1}}\left(\left(R_{1}-\phi^{*}\right) \gamma(s)\right)+q(s)
$$

(d). there exists $\theta>0$, such that $f_{\theta} \Delta(\gamma, \theta)>1$.

Then, the BVP (1) admits a positive solution.

Proof. Clearly, Condition (a) of Theorem 3.1 is satisfied for $\alpha=\alpha_{1}$. We have to prove that Condition (b) is also satisfied. Let $\varepsilon>0$ be such that $\left(f_{\theta}-\varepsilon\right) \Delta(\gamma, \theta)>1$. There exists $R_{\infty}$ such that $f\left(t, \frac{w}{p(t)}\right)>\left(f_{\theta}-\varepsilon\right) w$ for all $t \in[-\theta, \theta]$ and all $w \geq R_{\infty}$. Let

$$
R_{2}=1+\sup \left(R_{1}, \phi^{*}+\frac{R_{\infty}}{\gamma_{\sigma}}, \frac{\phi^{*}\left(f_{\theta}-\varepsilon\right) \Delta(\gamma, \theta)}{\left(f_{\theta}-\varepsilon\right) \Delta(\gamma, \theta)-1}\right)
$$

and

$$
\beta(t)=\left(f_{\theta}-\varepsilon\right)\left(R_{2}-\phi^{*}\right) \gamma(s)+q(s),
$$

where $\gamma_{\theta}=\min _{s \in[-\theta, \theta]} \gamma(s)$. Notice that

$$
\left(f_{\theta}-\varepsilon\right) \Delta(\gamma, \theta)\left(R_{2}-\phi^{*}\right)>R_{2} .
$$

We have then

$$
\Delta(\beta, \theta)=\sup _{t \in \mathbb{R}}\left(p(t) \int_{1 / \sigma}^{\sigma} G(t, s)\left(\left(f_{\theta}-\varepsilon\right)\left(R_{2}-\phi^{*}\right) \gamma(s)+q(s)\right) d s\right) \geq\left(f_{\theta}-\varepsilon\right) \Delta(\gamma, \theta)\left(R_{2}-\phi^{*}\right)>R_{2} .
$$

This completes the proof. 


\section{Example}

In this example, we consider the case of the BVP (1) when

$$
f(t, u)=e^{-\alpha|t|}(p(t) u)^{\beta}+c e^{-\alpha|t|} \frac{p(t) u}{1+p(t) u}-e^{-2 \alpha|t|}
$$

with $\beta<0$ and $\alpha, c>0$. We obtain from Theorem 3.1 the following corollary.

Corollary 4.1. Assume that $k<-r_{1}, l<r_{2}$,

$$
\alpha>\max \left(\frac{r_{2}}{2}, r_{1}-2 \beta r_{2}, \max \left(k,-r_{2}\right)-2 \beta r_{2}, \max \left(l,-r_{2}\right)+\left(r_{1}-r_{2}\right) \beta\right)
$$

and $c \Delta\left(\beta_{0}, \theta\right)>1+\phi^{*}$ for some $\theta>0$ where

$$
\beta_{0}(t)=\frac{\gamma(t)}{1+\gamma(t)}
$$

Then the BVP (1) within $f$ given in (5), has a positive solution.

Proof. We have to show that all the assumptions of Theorem 3.1 are satisfied. We have

$$
f\left(t, \frac{w}{p(t)}\right)=e^{-\alpha|t|}\left(w^{\beta}+\frac{c w}{1+w}-e^{-\alpha|t|}\right),
$$

leading to

$$
\left|f\left(t, \frac{w}{p(t)}\right)\right| \leq e^{-\alpha|t|}\left(w^{\beta}+c+1\right) .
$$

Set $q(t)=e^{-2 \alpha|t|}$ and for all $\rho>0$

$$
\omega_{\rho}(t)=e^{-\alpha|t|}, \quad \Psi_{\rho}(w)=w^{\beta}+c+1
$$

We have

$$
\int_{-\infty}^{+\infty} q(t) \eta(t) d t=\int_{-\infty}^{0} \exp \left(\left(2 \alpha-r_{2}\right) t\right) d t+\int_{0}^{+\infty} \exp \left(-\left(2 \alpha+r_{1}\right) t\right) d t
$$

Since (6) gives

$$
\alpha>\max \left(\frac{r_{2}}{2},-\frac{r_{1}}{2}\right)=\frac{r_{2}}{2},
$$

we obtain

$$
\int_{-\infty}^{+\infty} q(t) \eta(t) d t<\infty
$$

It is easy to see $\lim _{|t| \rightarrow \infty} p(t) q(t)=0$. Hence Hypothesis (2) holds. Since

$$
(\gamma(s))^{\beta}=\left\{\begin{array}{l}
\exp \left(2 \beta r_{2} t\right), \text { if } t \leq 0, \\
\exp \left(\beta\left(r_{1}-r_{2}\right) t\right), \text { if } t \geq 0,
\end{array}\right.
$$

we have for $t<0$,

$$
q_{-}(s) \omega_{\rho}(s) \psi_{\rho}(r \gamma(s))=(1+c) \exp \left(\left(-\max \left(k,-r_{2}\right)+\alpha\right) t\right)+r^{\beta} \exp \left(\left(\max \left(k,-r_{2}\right)+\alpha+2 \beta r_{2}\right) t\right)
$$

and for $t>0$

$$
q_{+}(s) \omega_{\rho}(s) \psi_{\rho}(r \gamma(s))=(1+c) \exp \left(\left(\max \left(l,-r_{2}\right)-\alpha\right) t\right)+r^{\beta} \exp \left(\left(\max \left(l,-r_{2}\right)-\alpha+2 \beta\left(r_{1}-r_{2}\right)\right) t\right) .
$$

Since (6) leads to

$$
\begin{aligned}
& \max \left(k,-r_{2}\right)+\alpha>0, \quad-\max \left(k,-r_{2}\right)+\alpha+2 \beta r_{2}>0, \\
& \max \left(l,-r_{2}\right)-\alpha<0, \quad \max \left(l,-r_{2}\right)-\alpha+2 \beta\left(r_{1}-r_{2}\right)<0,
\end{aligned}
$$

we obtain that

$$
\lim _{t \rightarrow-\infty} q_{-}(s) \omega_{\rho}(s) \psi_{\rho}(r \gamma(s))=\lim _{t \rightarrow+\infty} q_{+}(s) \omega_{\rho}(s) \psi_{\rho}(r \gamma(s))=0 .
$$

Also, we have

$$
\begin{aligned}
\int_{-\infty}^{+\infty} \delta(s) \omega_{\rho}(s) \psi_{\rho}(r \gamma(s)) d s= & (c+1) \int_{-\infty}^{0} \exp \left(\left(\alpha-r_{1}\right) s\right) d s+(c+1) \int_{0}^{+\infty} \exp \left(-\left(\alpha+r_{2}\right) s\right) d s \\
& +r^{\beta} \int_{-\infty}^{0} \exp \left(\left(\alpha-r_{1}+2 \beta r_{2}\right) s\right) d s+r^{\beta} \int_{0}^{+\infty} \exp \left(\left(-\alpha-r_{2}+\left(r_{1}-r_{2}\right) \beta\right) s\right) d s
\end{aligned}
$$


and since (6) leads to

$$
\alpha-r_{1}+2 \beta r_{2}>0, \quad-\alpha-r_{2}+\left(r_{1}-r_{2}\right) \beta<0,
$$

we arrive at

$$
\int_{-\infty}^{+\infty} \delta(s) \omega_{\rho}(s) \psi_{\rho}(r \gamma(s)) d s<\infty .
$$

Therefore, Hypothesis (3) is fulfilled. Now, for

$$
a_{R}(t)=e^{-\alpha|t|}\left(\left(R-\phi^{*}\right)^{\beta}(\gamma(t))^{\beta}+c+1\right)+e^{-2 \alpha|t|}
$$

straightforward computations lead to

$$
\Gamma\left(a_{R}\right) \leq \Lambda(R):=\lambda_{1}\left(R-\phi^{*}\right)^{\beta}+\lambda_{2},
$$

where

$$
\begin{aligned}
& \lambda_{1}=\sup _{t \in \mathbb{R}}\left(p(t) \int_{-\infty}^{+\infty} G(t, s) e^{-\alpha|s|}(\gamma(s))^{\beta} d s\right), \\
& \lambda_{2}=\sup _{t \in \mathbb{R}}\left(p(t) \int_{-\infty}^{+\infty} G(t, s)\left(e^{-\alpha|s|}(c+1)+e^{-2 \alpha|s|}\right) d s\right) .
\end{aligned}
$$

For large $R_{1}$, we have

$$
\Gamma\left(a_{R_{1}}\right) \leq \Lambda\left(R_{1}\right)<R_{1}
$$

and Condition (a) in Theorem 3.1 is satisfied. Now, for all $u \in P$ with $R=\|u\|>\phi^{*}$ and $t \in[-\theta, \theta]$ we have

$$
\begin{aligned}
f(t, u(t)-\phi(t))+q(t) & \geq c e^{-\alpha|t|} \frac{p(t)(u(t)-\phi(t))}{1+p(t)(u(t)-\phi(t))} \\
& \geq c e^{-\alpha|t|} \frac{\left(\|u\|-\phi^{*}\right) \widetilde{\gamma}(t)}{1+\left(\|u\|-\phi^{*}\right) \widetilde{\gamma}(t)}
\end{aligned}
$$

and we take

$$
\beta_{1}(t)=c e^{-\alpha|t|} \frac{\widetilde{\gamma}(t)}{1+\widetilde{\gamma}(t)}=c \beta_{0}(t)
$$

Therefore, for $R=1+\phi^{*}$, it holds that

$$
\Delta\left(\beta_{1}, \theta\right)=c \Delta\left(\beta_{0}, \theta\right)>1+\phi^{*}=R
$$

and Condition (b) of Theorem 3.1 is satisfied.

\section{References}

[1] R. P. Agarwal, D. O'Regan, Infinite Interval Problems for Differential, Difference and Integral Equations, Kluwer Academic Publisher, Dordrecht, 2001.

[2] O. R. Aris, The Mathematical Theory of Diffusion and Reaction in Permeable Catalysts, Clarendon, Oxford, 1975.

[3] C. Corduneanu, Integral Equations and Stability of Feedback Systems, Academic Press, New York, 1973.

[4] S. Djebali, K. Mebarki, Mutiple positive solutions for singular multi-point boundary value problem with general growth on the positive half-line, Electron. J. Differential Equations 2011 (2011) \#32.

[5] S. Djebali, O. Saifi, Positive solutions for singular BVPs with sign changing and derivative depending nonlinearity on the half-line, Acta Appl. Math . 110 (2010) 639-665.

[6] S. Djebali, O. Saifi, Positive solutions for singular BVPs on the positive half-line arising from epidemiology and combustion theory, Acta Math. Sci. 32 (2012) 672-694.

[7] S. Fu, K. Perera, On a class of semipositone problems with singular Trudinger-Moser nonlinearities, Discrete Contin. Dyn. Syst. Ser. S 14 (2021) $1747-1756$

[8] L. Lin, Y. Liu, D. Zhao, Multiple solutions for singular semipositone boundary value problems of fourth-order differential systems, Bound. Value Probl. 2021 (2021) \#79.

[9] J. D. Murray, Mathematical Biology I: An introduction, Springer-Verlag, New York, 2002.

[10] L. Sanchez, A note on a nonautonomous O.D.E. related to the Fisher equation, J. Comput. Appl. Math. 113 (2000) $201-209$.

[11] Y. Tian, W. Ge, Positive solutions for multi-point boundary value problem on the half-line, J. Math. Anal. Appl. 325 (2007) 1339-1349.

[12] Y. Tian, W. Ge, W. Shana, Positive solutions for three-point boundary value problem on the half-line, Comput. Math. Appl. 53 (2007) $1029-1039$. 